\title{
Análise por gênero da produção do corpo docente dos cursos de Economia nas Universidades Federais de Minas Gerais
}

\author{
Gender analysis of the production of economics professors at the Federal Universities of \\ Minas Gerais
}

\section{Resumo}

O presente trabalho analisa a produção do corpo docente dos cursos de Economia nas universidades públicas de Minas Gerais, em 2017. Para tanto, partese da verificação percentual dos professores dessa área, de acordo com o gênero. Ao estabelecer a hipótese de que há um número desproporcional de homens em relação às mulheres, busca-se, pela elaboração de um índice, medir a produção desses docentes levando em consideração a pesquisa (publicação em periódicos). Esse índice é criado com o intuito de concluir se existe indício de desigualdade de gênero no corpo docente de economia nas universidades em questão. Constatou-se que os homens representam $70 \%$ do total dos indivíduos estudados, e que a produção científica não foi um fator que justifica tal desproporção de gênero no corpo docente de Economia nas universidades federais de Minas Gerais.

Palavras-chave: Gênero; universidades federais de Minas Gerais; produção científica.

\begin{abstract}
The present study aims to analyze the scientific production of Economics professors at the public universities of Minas Gerais in 2017. For this purpose, the percentage of the professors in this area was verified, according to gender. By establishing the hypothesis that there is a disproportionate number of men in relation to women, the intention is to measure, through the elaboration of an index, the production of these professors considering their research (publication in periodicals). This index is created in order to conclude, after using the OLS, if there is evidence of gender inequality in the Economics courses at the analyzed universities. It was verified that men represent $70 \%$ of all the studied individuals, and that scientific production was not a factor that justifies such disproportion of gender in the teaching staff of Economics courses at the federal universities of Minas Gerais.
\end{abstract}

Keywords: Gender; federal universities of Minas Gerais; scientific production.

\footnotetext{
${ }^{1}$ Mestranda em Economia pela Universidade Federal de Viçosa. Bacharela em Economia pela Universidade Federal de Viçosa. Email: raniellasilva21@gmail.com

2 Doutor em Economia Aplicada pela Universidade Federal de Viçosa. Professor do Programa de Pós-Graduação em Economia da Universidade Federal de Viçosa. E-mail: jader.cirino@ufv.br
} 


\section{INTRODUÇÃO}

Atualmente, é perceptível a elevação da participação das mulheres no mercado de trabalho brasileiro, assim como a conquista destas por melhores condições de emprego. Segundo dados do Instituto Brasileiro de Geografia e Estatística (IBGE, 2015), as diferenças de inserção entre homens e mulheres foram reduzidas no período de 2003 a 2014, uma vez que as mulheres elevaram seu nível de ocupação de $40,5 \%$ para $45,4 \%$, enquanto os homens apresentaram expansão do nível de ocupação de $60,8 \%$ para $62,6 \%$.

A distribuição percentual da população ocupada, de acordo com o gênero, pode ainda ser pormenorizada segundo os grupamentos de atividades ${ }^{3}$. Embora a participação masculina seja majoritária à feminina em cinco grupamentos (indústria; construção; comércio; serviços prestados às empresas e outros serviços), tal superioridade apresentou queda no período de 2003 a 2014, com as mulheres aumentando sua representação em todas essas atividades econômicas, em especial no grupamento de "serviços prestados às empresas", no qual o aumento percentual das mulheres, para esse período, foi de 5,9 pontos percentuais.

No entanto, a ascensão das mulheres nos diversos setores da economia não ocorreu de forma igualitária, resultando, ainda nos dias atuais, em disparidades no que se refere à valorização e remuneração do emprego entre os gêneros. Tal fato é comprovado pelo IBGE (2015), quando expõe que a remuneração média do trabalho das mulheres, em 2014 , foi de apenas $74,2 \%$ do que recebiam os homens.

A expressão "teto de vidro" (glass ceiling) destaca-se como questão crucial para o entendimento dessa desigualdade de gênero no mercado de trabalho. Segundo Madalozzo (2011), tal denominação faz alusão à existência de barreira invisível que dificulta a progressão das mulheres para cargos de alto escalão.

Dessa forma, torna-se necessário discutir mais profundamente o fenômeno do glass ceiling ao se considerar que o nível educacional não é fator que explica as barreiras profissionais encontradas pelas mulheres, já que elas possuem a média de anos de estudo mais elevada (IBGE, 2014) e logram de maior sucesso acadêmico (INEP, 2007), comparativamente aos homens.

A concepção de "teto de vidro" vai ao encontro do exposto por Moschkovich e Almeida (2015), que ao estudarem sobre a questão da desigualdade de gênero na carreira acadêmica, em uma

${ }^{3}$ O IBGE (2014) define sete grupamentos de atividade, a saber: indústria; construção; comércio; serviços prestados às empresas; educação, saúde e administração pública; serviços domésticos; e outros serviços. 
universidade pública brasileira, abordaram o conceito de concentração horizontal e vertical no mercado de trabalho. $\mathrm{O}$ primeiro termo refere-se à presença desigual de determinado sexo em algumas áreas profissionais, enquanto o segundo reporta-se às circunstâncias em que, em uma mesma profissão, há desproporcional presença de homens ou mulheres em certo nível hierárquico. A pesquisa concluiu que, comparativamente aos homens, há indícios de que as docentes tendem a ter mais dificuldade em ingressar em algumas áreas e, na maioria dos casos, as professoras requerem mais tempo para alcançar o topo da carreira.

Nesse sentido, o presente trabalho pretende verificar a relação entre gênero e carreira acadêmica em uma universidade pública. Para tanto, realiza-se estudo da composição, de acordo com o gênero dos docentes, do curso de Ciências Econômicas nas universidades federais de Minas Gerais, bem como busca-se constatar se há relação entre o gênero e a produção científica desses professores. Especificamente, pretende-se: i) Elaborar um índice para as publicações dos docentes em periódicos Qualis ${ }^{4}$, nos anos de 2015 a 2017, de forma a medir a quantidade e qualidade de artigos científicos e, consequentemente, a produção desses profissionais; e ii) Verificar, pelo método de mínimos quadrados ordinários (MQO), a relação entre o índice de produtividade elaborado e o gênero dos docentes, controlando por algumas variáveis como: anos de carreira, classe acadêmica, participação em um programa de mestrado, contemplação com bolsa de produtividade nível 1 ou 2 , e instituição.

A relevância da pesquisa reside no fato de se analisar a questão de gênero em uma carreira de grande prestígio e influência intelectual e que, simultaneamente, está inserida em área caracterizada por ser majoritariamente masculina ${ }^{5}$. Vale ressaltar que, o estado de Minas Gerais foi escolhido para a realização da pesquisa em virtude de ser o estado brasileiro com maior número de universidades federais (GOMES, 2016).

Quanto à afirmativa de que o magistério público federal é carreira de prestígio, tem-se como respaldo Birnfeld e Costa (2012), que discorrem que os professores do ensino superior têm sua alta valorização atribuída à atuação na ponta do processo científico, sendo, portanto, carreira de

\footnotetext{
${ }^{4}$ O Qualis CAPES se define como um procedimento utilizado pela Coordenação de Aperfeiçoamento de Pessoal de Nível Superior (Capes), objetivando estratificar a produção intelectual dos programas de pós-graduação.

5 Segundo dados do INEP (2007), em 14 dos 26 estados e Distrito Federal brasileiros, o curso de Economia no ano de 2000 foi classificado como um dos 10 maiores cursos por matrícula. A partir da análise desses dados, percebe-se que, para esse curso, apenas Amapá apresentou mais ingressantes do sexo feminino. Em Roraima observou-se uma divisão igual entre os gêneros. Os demais estados exibiram maior porcentagem de matrículas masculinas, e, no Acre, a diferença entre os sexos chega a $31,1 \%$. Ademais, tal estatística é similarmente verificada em 2013, quando o site "O Globo" apontou, baseado nos dados do IBGE, que as mulheres eram minoria em apenas cinco cursos, sendo Economia um deles.
} 
responsabilidade na formação do quadro científico e técnico do país. Além disso, as universidades usufruem de autonomia didático-científica e administrativa, implicando que os docentes não trabalham sob pressão, mas sim orientados pela livre iniciativa.

Desse modo, ao se analisar a carreira com essas duas características (que seja de prestígio e com predominância masculina), se for constatado que há número desproporcional de indivíduos de determinado sexo, poderá ser indício de concentração horizontal, ainda mais se o gênero não for uma variável que influencia a produção cientifica dos docentes de Economia.

Vale esclarecer que, segundo a Lei $\mathrm{n}^{\circ} 12.772$ de 2012, os Cargos de Magistério Federal têm como atribuições o ensino, pesquisa, extensão, além do exercício de direção. Dessa forma, o presente trabalho avaliou a produção dos docentes apenas no que se refere à pesquisa, limitandose, ainda, às publicações em periódicos, para o período de 2015 a 2017 . Optou-se por focar nessa competência pela importância de se ter uma base científica consolidada para que o país alcance o desenvolvimento (FAGERBERG, SRHOLEC e VERSPAGEN, 2009).

Ademais, destaca-se que o presente trabalho busca contribuir com o debate acerca da relação entre produção científica e o gênero dos indivíduos, tendo em vista que não há na literatura consenso a respeito dessa temática (LETA e LEWISON, 2003; KYVIK e TEIGEN, 1996; e COLE e ZUCKERMAN, 1984).

Estudos como o aqui realizado, que buscam salientar as conquistas ou obstáculos das mulheres no mercado de trabalho, tornam-se objeto de grande significância, uma vez que tendem a servir de base para a formulação de políticas públicas que visam a igualdade de gênero.

\section{ASPECTOS TEÓRICOS E EMPÍRICOS SOBRE DESIGUALDADE DE GÊNERO}

No Brasil, o termo "gênero" foi introduzido no final dos anos 1980 ao debate relativo às mulheres. Esse termo contrapõe o paradigma do patriarcado ao elucidar a diferença entre o papel social e o biológico, não havendo, portanto, funções sociais rígidas para homens e mulheres. Nesse sentido, o gênero expressa a relação socialmente estabelecida entre os sexos (SANTOS e IZUMINO, 2005).

A investigação científica acerca da desigualdade de gênero ganhou grande relevância com o passar dos anos, sendo temática especialmente explorada pelo campo das Ciências Humanas, das Sociais e Sociais Aplicadas. Destaca-se, dentro deste último grupo, a subárea denominada 
Economia da Discriminação, que desenvolve modelos teóricos e empíricos, a fim de investigar as fontes de discriminação econômica (LOUREIRO, 2003).

Como definido por Loureiro (2003), pode se verificar a discriminação econômica de vários tipos $^{6}$, sendo, no entanto, a discriminação no mercado de trabalho, a forma mais estudada pela literatura. Pode-se classificar esse tipo de discriminação em duas categorias: (i) a discriminação direta, conhecida como "postmarket discrimination"; e (ii) a discriminação indireta, denominada de "premarket discrimation". Como as próprias denominações sugerem, a primeira categoria engloba as formas de discriminação que os indivíduos se deparam quando já estão inseridos no mercado de trabalho. Dessa forma, pode-se ressaltar três tipos de desigualdade: (i) a salarial - a questão de gênero influencia, fazendo com que haja diferença na remuneração, pois as mulheres recebem menos; (ii) de emprego - o mesmo indivíduo definido anteriormente apresenta desvantagem no que tange a oferta de emprego; e (iii) ocupacional - mulheres mesmo sendo capazes, não conseguem ocupar determinados cargos, em que predominam homens.

Já a segunda categoria representa o tipo de discriminação decorrente de desiguais oportunidades de se obter maior nível educacional ou treinamento (capital humano), sendo, portanto, discriminação enfrentada por mulheres antes de entrarem no mercado de trabalho.

As teorias desenvolvidas para se compreender sobre a discriminação e, em especial, para se elucidar como ela continua presente ao longo dos anos - mesmo sendo ineficiente e estando inserida em mercado competitivo - também podem ser divididas em duas linhas: o modelo teórico de discriminação por preferência e o modelo de discriminação estatística.

Primeiramente, o modelo teórico de discriminação por preferência advém do trabalho seminal de Becker (1957). Nele, tem-se estruturado o fato de que a discriminação, tanto por raça, sexo e origem, explica os diferenciais salariais. Basicamente, o modelo estabelece que o indivíduo racional (empregador, empregado e consumidor) pode ter preferência por discriminar, precisando, no entanto, estar disposto a pagar diretamente por isso (salários diferenciados para que a mão de obra feminina seja substituída pela masculina) ou indiretamente (redução da renda por estar preferindo um grupo independente da produtividade, reduzindo, consequentemente, o lucro). Conclui-se, dessa maneira, que a discriminação é sempre ineficiente.

\footnotetext{
${ }^{6}$ A discriminação econômica pode se dar no mercado de trabalho, entre as vizinhanças, segregação profissional, entre outros
} (LOUREIRO, 2003). 
Por outro lado, como exposto pelo modelo de Phelpes (1972), a discriminação estatística é usualmente eficiente, uma vez que não há preferência por discriminar. Se faz nesses modelos a pressuposição de que o empregador não tem conhecimento perfeito sobre os indivíduos, sendo, ainda, dispendioso obter mais informações. Como não se tem comportamento discriminatório, ele empregará características como raça e gênero para constatar se, de fato, esses atributos conduzem a diferentes produtividades entre determinados grupos.

Complementarmente, há os modelos teóricos de discriminação que, contrariamente às teorias discutidas, não assumem a abordagem de mercados competitivos, mas sim adotam a hipótese de segmentação do mercado de trabalho (LIMA, 1980; DOERINGER e PIORE, 1985).

Como se observa, as teorias anteriormente discutidas retratam a discriminação direta. Roemer (1998), no entanto, acrescenta aos modelos de discriminação, a teoria acerca do premarket discrimation, postulando duas concepções sobre igualdade de oportunidade. A primeira se refere ao que o autor define como "level the playing field" que se traduz como "nivelar o terreno". Nessa concepção, Roemer (1998) discorre sobre a importância dos esforços que a sociedade deve desprender para garantir a igualdade de condições entre indivíduos que competem por cargos.

Nesse sentido, deve-se considerar o background familiar das pessoas, uma vez que este tem grande influência na formação individual, refletindo, portanto, sobre o desenvolvimento de características relevantes para futura admissão no mercado de trabalho. Para exemplificar tal ideia, cita-se a oferta da educação compensatória para crianças que se inserem em contexto social desfavorecido, a fim de que grande proporção delas adquira as habilidades necessárias para competir por futuros empregos com crianças que tiveram um background familiar favorável.

Já o segundo princípio é denominado por Roemer (1998) como "O princípio da não discriminação", estado em que, na competição por cargos na sociedade, todos os indivíduos que possuem as características relevantes para o cumprimento da obrigação do serviço almejado estejam incluídos no grupo de candidatos elegíveis. Dessa forma, o julgamento para a posse deve ser influenciado apenas pelos atributos pertinentes.

A aplicação típica do princípio da igualdade de condições vai, portanto, além da definição de não discriminação, posto que, se não houver equalização da educação, por exemplo, a não discriminação sozinha na competição por empregos não constituiria a provisão de oportunidades iguais (ROEMER, 1998).

A exposição de todos esses conceitos atrelados à discriminação evidencia que o presente trabalho define-se como aplicação da teoria da discriminação direta do tipo ocupacional de Loureiro 
(2003), tendo em vista que busca-se, a partir da análise da composição e produção dos docentes dos cursos de economia das universidades federais de MG, verificar se as mulheres, mesmo sendo capazes, encontram maiores barreiras para ocupar o cargo de docência no curso, onde há historicamente predominância dos homens.

Complementarmente, toma-se como base o princípio da não discriminação (ROEMER, 1998), de maneira a embasar a justificativa de que a posse de um cargo de docência deve ser influenciada apenas pelos atributos pertinentes, objetivando a eficiência (BECKER, 1957) na carreira científica.

Alguns trabalhos buscaram estudar essa relação entre gênero e a carreira científica (COLE e ZUCKERMAN, 1984; KYVIK e TEIGEN, 1996; VELHO e LÉON, 1998; LETA e LEWISON, 2003). O primeiro, Cole e Zuckerman (1984), a partir de amostra extraída da Dissertação de Doutorado Americana (ADD), estudaram 526 homens e mulheres cientistas, demonstrando que homens publicaram, em média, $54 \%$ a mais do que as mulheres.

Já Kyvik e Teigen (1996), utilizando-se de questionários aplicados no ano de 1992, analisaram os professores pertencentes a quatro universidades da Noruega. Como resultado, apontaram que há diferenças de produtividade entre homens e mulheres - na média, homens publicaram 6,9 artigos, enquanto as mulheres publicaram 5,6 artigos durante os três anos estudados. Ainda, salientou-se duas variáveis que têm maior efeito sobre as diferenças de produtividade entre homens e mulheres: "crianças pequenas" e "colaboração em pesquisa".

No entanto, Leta e Lewison (2003), ao pesquisarem sobre a produção de 446 cientistas no Brasil, para o período de 1997-2001, constataram resultados diferentes aos apresentados anteriormente; isso porque, ao utilizar-se de informações advindas do Instituto de Informação Científica e do Censo dos Cientistas brasileiros ativo em 2000, concluiu-se que a participação das mulheres nas publicações científicas era similar às suas presenças percentuais nas áreas estudadas.

Ainda, Velho e Léon (1998), ao estudarem a inserção das mulheres em quatro unidades ${ }^{7}$ da Universidade Estadual de Campinas, bem como a contribuição delas para a produção científica referente a um período de oito anos (1986-1993), concluíram que, nos institutos que possuem maior presença de mulheres, as mulheres tendem a ser menos produtivas do que os homens, ao passo

\footnotetext{
${ }^{7}$ As quatro unidades estudadas foram: Instituto de Física (IF), Instituto de Química (IQ), Instituto de Biologia (IB) e Instituto de Ciências Humanas e Sociais (IFCH).
} 
que, quando analisa-se os institutos na qual há menor presença feminina, elas são tão, ou até mais, produtivas quanto os homens.

\section{MATERIAL E MÉTODOS: MODELO ANALÍTICO E FONTE DE DADOS}

Para estimar os determinantes da produtividade científica dos docentes universitários de Minas Gerais, utiliza-se a regressão na qual a variável dependente se define como índice de produtividade dos professores das universidades selecionadas (Produtivid ${ }_{i}$ ), enquanto as variáveis explicativas são o gênero $\left(\operatorname{sexo}_{i}\right)$, anos de carreira $\left(\operatorname{anoscar}_{i}\right)$, participação em um programa de mestrado ( mestrado $_{i}$ ), se recebe bolsa produtividade nível 1 ou nível 2, do Conselho Nacional de Desenvolvimento Científico e Tecnológico (CNPq) (bolsanivel1 ${ }_{i}$ e bolsanivel2 $i$ ), classe acadêmica (classe $k i,(k=6 a 8))$ e instituição $\left(\right.$ institu $\left._{l i}(l=9 a 15)\right)$, sendo $u_{i}$ o termo de erro, $B_{j}(j=0 a 15)$, os parâmetros a serem estimados e, $i$ os 189 indivíduos do estudo, conforme demonstrado pela equação (1).

$$
\begin{aligned}
\text { Produtivid }_{i}= & \hat{\beta}_{0}+\hat{\beta}_{1} \text { sexo }_{i}+\hat{\beta}_{2} \text { anoscar }_{i}+\hat{\beta}_{3} \text { mestrado }_{i}+\hat{\beta}_{4} \text { bolsanivel }_{i}+ \\
& \hat{\beta}_{5} \text { bolsanivel }_{i}+\hat{\beta}_{k} \text { classe }_{k i}+\hat{\beta}_{l} \text { institu }_{l i}+u_{i}
\end{aligned}
$$

Para a construção do índice de qualidade, fez-se necessário atribuir pesos para as publicações dos docentes. Para tanto, valeu-se dos critérios de classificação Qualis-ensino, divulgado pela Coordenação de Aperfeiçoamento de Pessoal de Nível Superior (CAPES, 2015), nos quais os periódicos são ponderados com valores de 0 a 100 pontos. Entretanto, para facilitar a pesquisa em progresso, os artigos receberam os seguintes valores: $A 1=1$ ponto; $A 2=0,85, B 1=$ $0,70, \mathrm{~B} 2=0,55, \mathrm{~B} 3=0,40, \mathrm{~B} 4=0,25, \mathrm{~B} 5=0,10$ e $\mathrm{C}=0,05$. Ademais, as publicações vinculadas às revistas que ainda não foram classificadas na área de economia receberam o menor peso $(0,05)$.

Para a criação da dummy de classe acadêmica distribuiu-se os docentes em quatro grupos: assistente, adjunto $\left(\right.$ classe $\left._{6 i}\right)$, associado $\left(\right.$ classe $\left._{7 i}\right)$ e titular $\left(\right.$ classe $\left._{8 i}\right)$; estabelecendo o primeiro grupo como categoria base.

Ainda, para a variável de instituição criou-se dummies que foram nomeadas de acordo com a sigla da universidade, resultando, portanto, em sete dummies denominadas: UFU (institu $\left.u_{9 i}\right)$, UNIFAL $\left(\right.$ institu $\left._{10 i}\right)$, UFJF $\left(\right.$ institu $\left._{11 i}\right)$, UFSJ $\left(\right.$ institu $\left._{12 i}\right)$, UFOP $\left(\right.$ institu $\left._{13 i}\right)$, UFV $\left(\right.$ institu $\left._{14 i}\right)$ e 
UFVJM (institu $15 i$ ). Foi considerado como categoria base a UFMG, por seu destaque nacional e internacional, como pode ser verificado no trabalho de Santos (2015), no qual, ao analisar o desempenho das universidades brasileiras a partir de um ranking nacional e cinco rankings universitários internacionais ${ }^{8}$, observou que entre as universidades que contribuíram em pelo menos $1 \%$ da produção científica brasileira no período de 2003 a 2012, a UFMG ocupava a $6^{\circ}$ posição, sendo a única de Minas Gerais entre as 10 primeiras do ranking. Dessa forma, espera-se sinal negativo para essas dummies.

Adicionalmente, define-se uma variável dummy que representa o sexo do indivíduo; com valor 1 para os homens e 0 para as mulheres. A partir da revisão de literatura e da discussão a respeito dos trabalhos de Leta e Lewison (2003), Velho e Léon (1998), Kyvik e Teigen (1996) e Cole e Zuckerman (1984), não há sinal esperado para essa variável.

Posteriormente, cria-se também uma variável dummy que indica a presença do docente no programa stricto sensu, de maneira que, designa-se o valor 1 para caso o indivíduo compusesse o corpo docente de mestrado e 0 caso contrário. Tomando como base o manual do usuário disponibilizado pelo Ministério da Educação (2015), tem-se que a pós-graduação stricto sensu é o espaço da pesquisa e da produção de conhecimento, de forma que há articulação entre as linhas, orientações, disciplinas ministradas e produtos da pesquisa. Dessa forma, fica explícito que a institucionalização de um programa de mestrado fomenta a pesquisa, sendo então esperado que essa variável impacte positivamente o índice de produção.

Insere-se ao modelo duas variáveis dummies, no intuito de captar o efeito dos docentes receberem a bolsa produtividade nível 1 e nível 2 sobre o índice, respectivamente. Assim, para a primeira variável atribui-se valor 1 caso o docente fosse contemplado com a bolsa nível 1 e valor 0 caso contrário. De forma análoga, para a segunda variável, confere-se valor 1 se o docente fosse contemplado com a bolsa nível 2 e valor 0 caso contrário. Para ambas variáveis espera-se sinal positivo, já que o CNPq, ao conceder as bolsas de produtividade, objetiva a valorização da produção, sendo destinadas, então, aos pesquisadores que se destacam entre seus pares.

Ademais, supõe-se que os docentes que são beneficiados com a bolsa nível 1 terão, em média, índice de produção mais elevado do que os agraciados com a bolsa nível 2. Isso porque, este nível concentra-se apenas na produtividade do pesquisador, enfatizando os trabalhos

\footnotetext{
8 Os cinco rankings internacionais são: Academic Ranking of World Universities (ARWU), Times Higher Education (THE), QS World University Rankings (QS), National Taiwan University Ranking (NTU) e Leiden Ranking (LR); e o ranking nacional é definido como o Ranking Universitário Folha (RUF).
} 
publicados e orientações, enquanto aquele exige mais do docente, no sentido de cobrar que os pesquisadores tenham gradual inserção tanto nacional quanto internacional, bem como envolvimento em atividades de gestão científica (CNPq, 2015).

Já no que se refere aos anos de carreira dos docentes, analisa-se essa questão a partir da variável anoscar, representando o tempo em que o indivíduo está vinculado à instituição como professor. Para tal formulação da variável, utiliza-se o estudo de Kyvik e Teigen (1996), que, ao relacionar a idade dos indivíduos com a publicação científica, verificou relação negativa entre as variáveis. Dessa forma, como a idade está positivamente relacionada com os anos de carreira dos professores, espera-se que, para o presente trabalho, os anos de carreira apresente sinal negativo.

No que concerne à variável dummy, que representa a classe acadêmica do indivíduo, esperase que o coeficiente seja positivo, isso porque, como exposto por Kyvik e Teigen (1996), ao se estabelecer como a categoria de referência, os professores assistentes, tanto os homens quanto as mulheres tendem a aumentar a produção científica à medida que avançam nas classes acadêmicas.

Para estimar esse modelo, utiliza-se o Método de Mínimos Quadrados Ordinários (MQO). Segundo Gujarati e Porter (2011), o MQO caracteriza-se por ser o critério que eleva os resíduos ao quadrado, designando maior peso aos resíduos muito dispersos em relação à função de regressão amostral. Assim, esta é a menor possível, já que os resíduos recebem a importância de acordo com a proximidade das observações individuais em relação à função de regressão amostral. Portanto, o princípio de mínimos quadrados, para uma dada amostra, oferece estimativas únicas de $\beta_{1}$ e $\beta_{2}$ que asseguram o menor valor possível do somatório dos resíduos. Desta forma, sabendo das hipóteses do modelo clássico de regressão linear, as estimativas de MQO têm propriedades ótimas, conhecidas como MELNV (propriedade de melhor estimador linear não viesado).

Para constatar que não há relaxamento das hipóteses do modelo clássico, o que pode afetar a característica dos estimadores de MQO de serem MELNV, foi realizado o Teste de Breusch-Pagan para verificar a homocedasticidade, ou seja, se as variâncias dos erros são iguais. Ainda, aplicouse o Fator de Inflação Variável (VIF), buscando constatar a presença de multicolinearidade. Sabese que, quando o VIF >10 tem-se multicolinearidade prejudicial.

Em primeiro momento, o trabalho utiliza-se dos dados disponibilizado pelas 11 universidades federais de Minas Gerais ${ }^{9}$. Após realizar a filtragem dessas instituições, levando em consideração

\footnotetext{
${ }^{9}$ As onze universidades federais de Minas Gerais são: UNIFAL, UNIFEI, UFJF, UFLA, UFMG, UFOP, UFSJ, UFU, UFV, UFTM, UFVJM.
} 
quais possuem o curso de Ciências Econômicas, restaram oito, sendo excluídas, portanto, a Universidade Federal de Itajubá, a de Lavras e a do Triângulo Mineiro.

No segundo momento, consultou-se a Plataforma Lattes, disponibilizada pelo Conselho Nacional de Desenvolvimento Científico Tecnológico (CNPq), buscando para cada indivíduo em análise, o número de publicações, assim como a revista em que foram vinculados os artigos, referentes ao período de 2015 a $2017^{10}$. Ainda, a partir do currículo Lattes foram listados também os anos de carreira na instituição, bem como a classe acadêmica do indivíduo e, ainda, se este é contemplado com alguma bolsa de produtividade do CNPq.

\section{RESULTADOS E DISCUSSÃO}

\section{Corpo docente do curso de Ciências Econômicas em Minas Gerais}

O corpo docente de Ciências Econômicas em Minas Gerais é composto de 189 professores, distribuídos em oito universidades federais. A TAB. 1 apresenta o número total de professores do curso de Economia, bem como suas distribuições de acordo com o gênero e instituição.

Destaca-se que, desse total de professores, $70,3 \%$ são homens, mostrando que o curso apresenta número desproporcional de docentes do sexo masculino em relação ao sexo feminino.

Considerando individualmente as universidades, verifica-se que tal proporção de gênero tem grande variabilidade entre as instituições, embora em nenhuma delas haja mais professoras. A universidade que apresenta maior proporção dessas últimas é a UNIFAL, onde as mulheres representam $41 \%$ do corpo docente.

Dessa maneira, constata-se que os cursos de Economia nas universidades federais de Minas Gerais se definem como caso de concentração horizontal, tendo em vista presença desigual de determinado sexo; o corpo docente da Universidade Federal de Viçosa se constitui o principal exemplo desse tipo de concentração.

\footnotetext{
10 Foram relacionadas publicações em periódicos de 2015 até 04/09/2017, buscando um maior número de observações, mas se limitando a um período mais recente.
} 
Tabela 1 - Constituição do corpo docente do curso de Economia - Minas Gerais, 2017

\begin{tabular}{cccccc}
\hline Universidade & $\begin{array}{c}\text { Total de } \\
\text { Professores }\end{array}$ & $\begin{array}{c}\text { Número de } \\
\text { mulheres }\end{array}$ & \% Mulheres & $\begin{array}{c}\text { Número de } \\
\text { Homens }\end{array}$ & $\begin{array}{c}\% \\
\text { Homens }\end{array}$ \\
\hline UNIFAL & $22^{*}$ & 09 & 41 & 13 & 59 \\
UFJF & 24 & 07 & 29 & 17 & 71 \\
UFMG & 41 & 12 & 29 & 29 & 71 \\
UFOP & 18 & 04 & 22 & 14 & 72 \\
UFSJ & 18 & 05 & 28 & 13 & 68 \\
UFU & $34^{* *}$ & 11 & 32 & 23 & 81 \\
UFV & 16 & 03 & 19 & 13 & 69 \\
UFVJM & 16 & 05 & 31 & 11 & 70 \\
\hline TOTAL & 189 & 56 & 30 & 133 & \\
\hline
\end{tabular}

Fonte: Resultados da pesquisa.

Nota: *Para obter esse número de professores, filtrou-se os docentes que têm formação em Ciências Econômicas, uma vez que o site da instituição disponibiliza todos os docentes pertencentes ao Instituto de Ciências Sociais Aplicadas (ICSA).

** Seguiu-se o mesmo processo anteriormente relatado, uma vez que o site da instituição disponibiliza todos os docentes pertencentes ao Instituto de Economia (IEUFU).

A TAB. 2 apresenta informações referentes às variáveis quantitativas do modelo econométrico. Observa-se que o índice de produtividade, construído a partir do número de publicações e seus respectivos pesos, de acordo com a revista em que foi vinculado, varia de 0 a 7,15, e os docentes, na média, apresentam produtividade de 1,27 para o período de 2015 a 2017. Ainda, o coeficiente de variação $(\mathrm{CV})$ para o índice apresenta alta dispersão dos valores $(\mathrm{CV}=$ $134,84 \%)$, indicando a heterogeneidade dos dados.

Tabela 2 - Variáveis quantitativas do modelo econométrico Minas Gerais, 2015-2017

\begin{tabular}{ccc}
\hline Estatísticas & Índice & Anos de carreira \\
\hline Média & 1,27 & 11,78 \\
Desvio Padrão & 1,71 & 10,16 \\
Variância & 2,92 & 103,23 \\
Mínimo & 0,00 & 0,00 \\
Máximo & 7,15 & 40,00 \\
\hline
\end{tabular}

Fonte: Resultados da pesquisa.

Quando se analisa os anos de carreira, observa-se, similarmente, tal heterogeneidade, uma vez que o coeficiente de variação é igual a $86,25 \%$. Tem-se ainda que os professores apresentam a média de 12 anos de carreira.

Ao estratificar o cargo de acordo com o gênero dos docentes (TAB. 3), a classe de maior nível hierárquico (titular) apresenta acentuada assimetria entre homens e mulheres, indicando possível 
caso de concentração vertical. Esse fato foi abordado por Léon e Velho (1998), que ao retratarem sobre a sub-representatividade das mulheres na carreira científica, expõem que essa participação ainda declina à medida que se avança os níveis de classe acadêmica.

Complementarmente, destaca-se que os dados demonstram que não há tendência de mudança da composição do corpo docente, no que tange ao gênero dos professores, já que a classe acadêmica de menor nível (assistente) reproduz a proporção de homens e mulheres verificado nas demais classes.

Tabela 3 - Classe acadêmica dos professores das universidades federais de acordo com o gênero - Minas Gerais, 2017

\begin{tabular}{cccccc}
\hline $\begin{array}{c}\text { Classe } \\
\text { Acadêmica }\end{array}$ & $\begin{array}{c}\text { Total de } \\
\text { Professores }\end{array}$ & $\begin{array}{c}\text { Número de } \\
\text { Mulheres }\end{array}$ & $\begin{array}{c}\text { \% de } \\
\text { Mulheres }\end{array}$ & $\begin{array}{c}\text { Número de } \\
\text { Homens }\end{array}$ & $\begin{array}{c}\text { \% de } \\
\text { Homens }\end{array}$ \\
\hline Assistente & 25 & 07 & 28,00 & 18 & 72,00 \\
Adjunto & 116 & 37 & 31,90 & 79 & 68,10 \\
Associado & 32 & 09 & 28,12 & 23 & 71,88 \\
Titular & 16 & 03 & 18,75 & 13 & 81,25 \\
\hline TOTAL & 189 & 56 & 29,63 & 133 & 70,37 \\
\hline
\end{tabular}

Fonte: Resultados da pesquisa.

Outras variáveis de controle que requerem maiores exposições referem-se às bolsas de produtividade.

Tabela 4 - Distribuição das Bolsas de produtividade nas universidades federais - Minas Gerais, 2017

\begin{tabular}{|c|c|c|c|c|c|c|}
\hline \multirow{3}{*}{ Universidade } & \multicolumn{4}{|c|}{ Bolsas de produtividade } & \multirow{3}{*}{$\begin{array}{c}\% \\
{ }^{*} \text { mulheres } \\
\text { bolsistas }\end{array}$} & \multirow{3}{*}{$\begin{array}{c}\% \\
\text { *homens } \\
\text { bolsistas }\end{array}$} \\
\hline & \multicolumn{2}{|c|}{ Nível 1} & \multicolumn{2}{|c|}{ Nível 2} & & \\
\hline & Feminino & Masculino & Feminino & Masculino & & \\
\hline UFMG & 01 & 02 & 03 & 07 & 33 & 31 \\
\hline UFJF & 00 & 01 & 03 & 04 & 43 & 29 \\
\hline UFU & 00 & 00 & 01 & 06 & 09 & 23 \\
\hline TOTAL & 01 & 03 & 07 & 17 & 27 & 29 \\
\hline
\end{tabular}

Fonte: Resultados da pesquisa.

Nota: * A porcentagem é calculada sobre o número de mulheres/homens contempladas com bolsa sobre o total de mulheres/homens no corpo docente.

Como pode ser observado na TAB. 4, há apenas três universidades que apresentam docentes contemplados com essas bonificações, totalizando 28 professores. Verifica-se que, desses, apenas quatro recebem bolsa nível 1, o que significa que aproximadamente $86 \%$ desses indivíduos são bonificados com a bolsa nível 2. Esse fato é compreensível, tendo em vista as exigências para se alcançar o nível mais elevado. Ademais, tem-se equivalência (27\% e 29\%) 
quando se realiza estudo relacionando a bolsa produtividade com o gênero, levando em consideração o total de mulheres e homens em cada corpo docente, o que já se constitui indício da não relação entre gênero e produção científica. Por fim, analisa-se nas tabelas 5 e 6 a variável dependente do presente estudo, ou seja, a produção científica total dos docentes do curso de Ciências Econômicas por universidade federal mineira, bem como o índice de produção de acordo com o gênero do docente.

Tabela 5 - Índice de produção dos docentes dos cursos de Economia por universidade federal - Minas Gerais, 2015-2017

\begin{tabular}{|c|c|c|c|c|c|c|c|c|c|c|}
\hline \multirow[b]{2}{*}{ Instituição } & \multicolumn{8}{|c|}{ Número de publicações de acordo com a classificação Qualis* } & \multirow[b]{2}{*}{ Índice } & \multirow[b]{2}{*}{$\begin{array}{l}\text { Índicel } n^{\circ} \\
\text { de docentes }\end{array}$} \\
\hline & $\begin{array}{l}\text { A1 } \\
\text { (1) }\end{array}$ & $\begin{array}{c}A 2 \\
(0,85)\end{array}$ & $\begin{array}{c}\text { B1 } \\
(0,7)\end{array}$ & $\begin{array}{c}\text { B2 } \\
(0,55)\end{array}$ & $\begin{array}{c}\text { B3 } \\
(0,4)\end{array}$ & $\begin{array}{c}\text { B4 } \\
(0,25)\end{array}$ & $\begin{array}{c}\text { B5 } \\
(0,1)\end{array}$ & $\underset{(0,05)}{C}$ & & \\
\hline UFMG & 12 & 8 & 56 & 29 & 6 & 11 & 12 & 37 & 82,00 & 2,00 \\
\hline UFJF & 6 & 4 & 37 & 16 & 3 & 4 & 6 & 3 & 47,05 & 1,96 \\
\hline UFV & 1 & 1 & 20 & 15 & 7 & 10 & 8 & 16 & 31,00 & 1,94 \\
\hline UFU & 3 & 5 & 36 & 21 & 19 & 4 & 24 & 18 & 55,55 & 1,62 \\
\hline UFSJ & 0 & 0 & 6 & 4 & 4 & 2 & 2 & 5 & 8,95 & 0,50 \\
\hline UFOP & 0 & 0 & 1 & 4 & 1 & 11 & 3 & 9 & 6,80 & 0,40 \\
\hline UNIFAL & 0 & 0 & 1 & 5 & 2 & 0 & 4 & 14 & 5,35 & 0,25 \\
\hline UFVJM & 1 & 0 & 0 & 0 & 3 & 2 & 1 & 5 & 3.05 & 0,19 \\
\hline
\end{tabular}

Fonte: Resultados da pesquisa.

Nota: *Entre parênteses está especificado o peso da revista científica.

Como pode-se observar, em termos absolutos, a UFMG é a instituição que apresenta o maior índice de produção, somando, portanto, 82,00 pontos, quando se considera a quantidade e a qualidade de suas publicações científicas. Tal constatação era esperada, uma vez que, com base no estudo de Santos (2015), acreditava-se que a UFMG ocuparia a primeira posição quando se considerasse a produção científica dos docentes, tendo em vista a sua grande notoriedade no cenário nacional e internacional.

Essa superioridade persiste quando analisa-se a produção por docente, no entanto, a diferença em relação às outras universidades se reduz. Pode-se estabelecer, em certa medida, homogeneidade do índice per capita entre UFMG, UFJF, UFV e UFU. Justifica-se esse fato tendo 
como base os anos de existência do curso de Economia nessas quatro instituições ${ }^{11}$, e que essas universidades apresentam programas de pós-graduação em Economia já consolidados ${ }^{12}$.

Adicionalmente, constatou-se que 32,3\% dos docentes do curso de Ciências Econômicas das universidades federais mineiras não publicaram artigos em periódicos, o que resulta no fato de que, 128 professores foram responsáveis pelas 548 publicações aqui estudadas.

A TAB. 6 complementa a análise do índice de produção ao incluir o gênero dos professores do curso de Ciências Econômicas.

Tabela 6 - Índice de produção por gênero dos docentes dos cursos de Economia das universidades federais Minas Gerais, 2015 -2017

\begin{tabular}{ccccc}
\hline Universidade & Índice Feminino & Índice $^{\circ} \mathbf{n}^{\mathbf{m}}$ mulheres & Índice Masculino & Índice/ $^{\circ}$ homens \\
\hline UFMG & 25,35 & 2,11 & 56,65 & 1,95 \\
UFJ & 15,90 & 2,27 & 31,15 & 1,83 \\
UFV & 4,50 & 1,50 & 26,50 & 2,04 \\
UFU & 17,8 & 1,62 & 37,75 & 1,64 \\
UFSJ & 4,70 & 0,94 & 4,25 & 0,33 \\
UFOP & 1,05 & 0,26 & 5,75 & 0,41 \\
UNIFAL & 0,20 & 0,02 & 5,15 & 0,40 \\
UFVJM & 0,75 & 0,15 & 2,30 & 0,21 \\
\hline
\end{tabular}

Fonte: Resultados da pesquisa.

Verifica-se, assim, que o resultado em geral vai ao encontro dos trabalhos de Kyvik e Teigen (1996) e, Cole e Zuckerman (1984), uma vez que, salvo o caso da UFSJ, nas demais universidades, as mulheres apresentam menor produção do que os homens. No entanto, assim como Leta e Lewison (2003) ressaltam, quando analisa-se a produção de acordo com a proporção da presença do gênero no campo estudado, mulheres são tão produtivas quanto os homens.

\section{Determinantes da produtividade dos professores do curso de Economia em Minas Gerais}

Após a identificação da heterogeneidade entre homens e mulheres na composição da docência das unidades federais mineiras e a constatação de que as produtividades de ambos são similares, procede-se com a estimação dos determinantes da produtividade dos professores do

\footnotetext{
11 O curso de Ciências Econômicas foi criado na UFJF, UFMG, UFU, UFV, UFSJ, UFVJM, UFOP, UNIFAL, no ano de 1943, 1945, 1962, 1976, 1976, 2006, 2009 e 2009, respectivamente. Dessa forma, nota-se que, com exceção da UFSJ, instituições que apresentam os cursos de Ciências Econômicas mais antigos, exibem maior índice por docente.

${ }^{12}$ Considera-se que a UFMG, UFU, UFJF e UFV apresentam programa de pós-graduação consolidado, uma vez que iniciaram suas atividades no ano de 1968, 1996, 2006 e 2006, respectivamente. Ao passo que, a UFOP instituiu seu programa em 2016 e, ainda mais recentemente, no ano de 2017, a UNIFAL inaugurou seu programa de pós-graduação em economia.
} 
curso de economia em Minas Gerais. Pode-se examinar, na TAB. 7, as estimativas produzidas para a regressão (1), obtidas pelo método de mínimos quadrados ordinários (MQO). Para se chegar ao resultado exposto, garantindo assim os melhores estimadores lineares não viesados, fez-se necessário aplicar o teste de Breusch-Pagan, o que resultou na constatação de que o modelo violou o pressuposto de homocedasticidade, sendo então fundamental corrigir esse problema a partir do erro padrão robusto. Quanto à presença de multicolinearidade, o Fator de Inflacionamento da Variância retornou o valor médio de 1,84, significando, portanto, que, em média, as variáveis explicativas são moderadamente correlacionadas.

Ademais, o modelo apresentou-se significativo a partir da análise do Teste $F$ (significância global) e, adicionalmente, exibiu coeficiente de determinação $\left(R^{2}\right)$ de 0,45 , o que indica que as variáveis independentes explicam, conjuntamente, $45 \%$ do índice de produção científica.

Tabela 7 - Modelo econométrico para o índice de produção científica - Minas Gerais, 20152017

\begin{tabular}{|c|c|c|c|}
\hline \multirow[b]{2}{*}{ Variáveis } & \multirow[b]{2}{*}{ Coeficientes } & \multicolumn{2}{|c|}{$\begin{array}{r}\text { Número de Observações }=189 \\
F(15,173)=8,56 \\
\text { Prob }>F=0,0000 \\
\text { R-squared }=0,4514\end{array}$} \\
\hline & & Desvio Padrão & Teste $t$ \\
\hline Constante & $1,2726^{*}$ & 0,3936 & 3,23 \\
\hline Sexo & $-0,0556$ & 0,2286 & $-0,24$ \\
\hline Anoscar & $-0,0384^{*}$ & 0,0115 & $-3,32$ \\
\hline Mestrado & $1,2059^{*}$ & 0,3062 & 3,94 \\
\hline Bolsanivel1 & $2,1502^{* * *}$ & 1,2929 & 1,66 \\
\hline Bolsanivel2 & $0,9381^{* *}$ & 0,4577 & 2,05 \\
\hline Adjunto & 0,1463 & 0,1459 & 1,00 \\
\hline Associado & 0,4842 & 0,3522 & 1,37 \\
\hline Titular & $1,0967^{* * *}$ & 0,5877 & 1,87 \\
\hline UFU & $-0,1651$ & 0,3712 & $-0,44$ \\
\hline UNIFAL & $-0,8917^{* *}$ & 0,3581 & $-2,49$ \\
\hline UFJF & 0,1317 & 0,4379 & $-0,30$ \\
\hline UFSJ & $-0,6219^{* * *}$ & 0,3558 & $-1,75$ \\
\hline UFOP & $-1,1837$ * & 0,3945 & $-3,00$ \\
\hline UFV & 0,0918 & 0,4668 & $-0,20$ \\
\hline UFVJM & $-0,8973^{* *}$ & 0,3604 & $-2,49$ \\
\hline
\end{tabular}

Fonte: Resultados da pesquisa.

Nota: *Significativo a $1 \%$.

**Significativo a $5 \%$.

${ }^{* * *}$ Significativo a $10 \%$. 
Posto isso, tem-se que o coeficiente da variável referente aos anos de carreira dos docentes - anoscar - foi significativo a 1\%. Dessa forma, o sinal esperado para o coeficiente foi confirmado, indo, portanto, ao encontro do estudo de Kyvik e Teigen (1996), possibilitando, assim, concluir que os anos de carreira impactam negativamente na produção dos professores do curso de Ciências Econômicas das universidades federais mineiras. Pode-se justificar esse fato tendo em vista que os docentes não têm dedicação exclusiva para pesquisa, mas são encarregados também de atividades referentes ao ensino e à extensão. No entanto, vale destacar que esse impacto é quase nulo, já que um ano a mais de carreira diminui em 0,03 pontos o índice de produção dos docentes, ou seja, tomando como base o índice médio e máximo, a cada ano, os docentes reduziriam seus índices de produção em apenas $2 \%$ e $0,4 \%$, respectivamente.

A variável representativa dos indivíduos que se inserem em um programa stricto sensu é positiva e significativa a 1\%, confirmando as expectativas de que participar de um programa de mestrado faz com que os docentes apresentem, em média, índice de produção 1,2 pontos superior aos docentes que não se envolvem com essa atividade. Tal constatação é plausível, considerando que os docentes que compõem programa de mestrado tendem a usufruir de maior acesso ao que se pode definir como inputs da pesquisa, ou seja, além das orientações de monografias advindas da graduação, esses docentes, em especial, também auxiliam nas elaborações de dissertações, e, em alguns casos, ajudam na produção de teses, apresentando, então, maior leque de trabalhos que podem se converter em artigos científicos. Analisando as dummies bolsas de produtividade nível 1 e nível 2, tem-se que ambas são positivas e significativas a 10\% e 5\%, respectivamente, e os docentes que são beneficiados com a bolsa nível 1, produzem em média, expressivamente mais (2,15 pontos) em comparação aos docentes quem não são contemplados com essa bolsa. Já os indivíduos agraciados com a bolsa nível 2 também apresentam índice de produção mais elevado em relação aos que não possuem essa bonificação (em média 0,94 pontos).

Justifica-se tal constatação considerando-se a discussão realizada por Wainer e Vieira (2013), tendo em vista que, ao mesmo tempo que o objetivo da bolsa produtividade é premiar cientistas de qualidade, também busca-se com esse bônus incentivar a continuidade da produção científica desses pesquisadores. Dessa forma, docentes que recebem alguma dessas bolsas já tendem a apresentar notoriedade no campo científico, comparativamente aos seus pares, e, ainda, precisam manter a produção de qualidade para continuar a receber essa bonificação. Há, entretanto, grande distância entre os dois níveis, tanto em termos de exigências quanto em benefícios. 
Quando se examina as variáveis referentes à classe acadêmica, tem-se que as dummies Adjunto e Associado não foram significativas, ao passo que titular mostrou-se significativa a $10 \%$. Verificou-se, então, o sinal esperado apenas para essa última categoria, o que implica dizer que os professores pertencentes à classe titular do curso de Economia das universidades federais de Minas Gerais, exibem, em média, índice de produção 1,1 ponto maior do que os professores assistentes dessa área. A explicação para esse resultado apoia-se no fato de que, segundo o Ministério da Educação (2012), essa é a única classe que exige do docente conquistar a aprovação de memorial, exigindo então excelência nas atividades de ensino, pesquisa, extensão, gestão acadêmica e produção profissional relevante.

No que concerne às variáveis de instituição, obteve-se alguns resultados inesperados. Isso porque, supunha-se, como retratado por Santos (2015), que o papel de destaque da UFMG nos rankings das universidades, bem como na colaboração das publicações científicas brasileiras, faria com que as demais instituições analisadas no presente estudo apresentassem o sinal negativo para os seus respectivos coeficientes. Essa expectativa foi verificada para UNIFAL, UFSJ, UFOP e UFVJM. Entretanto, para UFU, UFJF e UFV os coeficientes não foram significativos, implicando que não há diferença de publicação, quando analisa-se a quantidade e qualidade entre os professores do curso de Ciências Econômicas destas universidades comparativamente aos professores desse mesmo curso da UFMG.

Explica-se essa situação baseado no exposto pelo Instituto de Pesquisa Econômica Aplicada (IPEA, 2003), que relata que os centros e as instituições de pesquisas tendem a ser heterogêneos no que tange à organização e características relevantes em termos de capacidade de produção de conhecimento, podendo se diferenciar pelo tamanho da equipe de pesquisa, bem como qualidade e prestígio da universidade. Assim, pode-se entender que a UFJF, UFV, UFU e UFMG tendem a ser mais homogêneas no que se refere a estrutura da instituição, permitindo, assim, supor que o suporte da universidade tende a impactar nas publicações dos docentes.

Por fim, a principal variável de interesse, "sexo", que não havia sinal esperado para o seu coeficiente, não se apresentou significativa, o que resulta na conclusão de que não há diferença na produção científica entre os professores do curso de Economia das universidades federais mineiras, quando se analisa o gênero desses docentes. Esse resultado é semelhante ao exposto por Leta e Lewison (2003) e, em certa medida, é respaldado por Velho e Léon (1998), sendo, dessa maneira, divergente dos trabalhos de Cole e Zuckerman (1984) e Kyvik e Teigen (1996), obras já amplamente discutidas. 
A exposição dos resultados e a discussão desenvolvida comprovam, empiricamente, que o gênero não se constitui variável que influencia na produção científica dos docentes do curso de Ciências Econômicas das universidades federais mineiras. Se mulheres são tão produtivas quanto homens nessa área, a discriminação de gênero surge como possível explicação para que $70 \%$ dos docentes sejam homens. Esse indício de discriminação pode ser preocupante, dado que, pode-se estar deixando de contratar indivíduos de maior produtividade, incorrendo na perda de eficiência em área importante para o desenvolvimento do país, tanto diretamente quanto indiretamente, ao influenciar na formação do quadro científico brasileiro.

Outra constatação preocupante refere-se à concentração vertical nessa área, ou seja, a possível existência de barreira que impede as docentes de alcançarem a classe de titular. Variável como a dupla jornada de trabalho (produtivo e reprodutivo) das mulheres, pode se constituir explicação para esse fenômeno de glass ceiling. Ressalta-se que os resultados apontam para a continuidade dessa concentração horizontal e vertical no curso de Economia.

No entanto, o presente trabalho, por ser muito específico ao medir a produtividade dos professores apenas por sua função de pesquisa e, ainda mais, restringir à análise das publicações em periódicos, torna-se impossível dizer que o indício de discriminação é de fato realidade. Para realizar tal afirmação, seria necessário obter mais informações sobre a ponderação de currículo e desempenho dos candidatos homens e mulheres para o cargo de professor efetivo, bem como a proporção de cada sexo na disputa por uma vaga, tendo em vista que a discriminação pode estar no processo de formação ou no interesse histórico pela área. Fundamental também seria inserir ao estudo, a produção dos docentes dessa área em termos de atividade de ensino, extensão e direção.

\section{CONCLUSÕES}

O presente estudo partiu da hipótese que existe número desproporcional de docentes homens em relação às mulheres nos cursos de Ciências Econômicas das universidades federais de Minas Gerais. Assim, propõe-se medir a produtividade desses docentes a partir da atividade relacionada com a pesquisa (publicações em periódicos), de modo a averiguar se o gênero seria fator que diferencia a produção científica, o que justificaria a heterogeneidade hipoteticamente citada. 
Para tanto, em primeiro momento utilizou-se de análise percentual do total de professores universitários do curso, concluindo assim que os homens representam $70 \%$ do total desses indivíduos.

Consequentemente, pela utilização do método de mínimos quadrados ordinários (MQO), regrediu-se o índice de produtividade em relação às variáveis anos de carreira, classe acadêmica, participação em programa de mestrado, contemplação com bolsa de produtividade nível 1 ou 2 , e instituição; a primeira apresentou-se não significativa, resultando assim que homens e mulheres pertencentes ao grupo pesquisado, não apresentam diferença na produção científica.

Dessa forma, constatou-se que há indício de desigualdade de gênero no corpo docente dos cursos de Economia das universidades federias de Minas Gerais. Entretanto, não se pode afirmar que essa situação é caso confirmado de desigualdade de gênero, posto que, mesmo que a produção científica não tenha sido determinante para explicar a desproporção de homens e mulheres no campo estudado, outros fatores podem influenciar nesse desequilíbrio de gênero, como a própria escolha dos indivíduos em ingressar no curso de Ciências Econômicas, bem como sua persistência na pós-graduação, até a prestação do concurso público. Assim, seria necessário analisar a proporção de mulheres e homens desde a graduação, de forma a expandir a discussão a respeito da composição do corpo docente dos cursos de Economia das universidades federais de Minas Gerais, quando se leva em consideração o gênero dos professores.

O presente trabalho também apresenta algumas limitações, como, ao pesquisar a produção dos docentes, focou-se apenas em uma área de atuação, não analisando a produção dos docentes no que se refere à atividade de extensão, ensino e administração; sendo interessante, portanto, incorporar tais investigações a futuros trabalhos. Ademais, há mais variáveis que podem ser inseridas de forma a obter melhor ajustamento do modelo - como uma que capte a rede informal dos indivíduos, bem como variável que mensure o impacto de se ter filhos pequenos - que não foram incorporadas no trabalho por não estarem disponíveis, sendo necessário a aplicação de questionários.

Apesar dessas limitações, tem-se que os trabalhos como o aqui realizado são de grande relevância, uma vez que buscam contribuir para a literatura que aborda a temática a respeito da relação entre produção científica e gênero, bem como auxilia a direcionar políticas públicas para áreas onde minorias não conseguiram grande inserção, restando constatar se tais características, por si só, constituem barreiras à incorporação desses indivíduos no mercado de trabalho. 


\section{REFERÊNCIAS}

BECKER, Gary Stanley. The economics of discrimination. University of Chicago Press. Chicago, IL, 1957.

BIRNFELD, Carlos Andre Hüning; COSTA, Eder Dion de Paula. A carreira docente federal do ensino superior brasileiro e suas inconstitucionalidades - 25 anos de incertezas. Repositório Institucional da Universidade Federal do Rio Grande (RI FURG), 2012. Acesso em: 08/11/2016. [Visualizar] Google

BRASIL. Lei $N^{\circ}$ 12.772, de 28 de dezembro de 2012. Dispõe sobre a estruturação do Plano de Carreiras e Cargos de Magistério Federal; sobre a Carreira do Magistério Superior. Acesso em: 06/10/2016. [Visualizar]

COLE, Jonathan; ZUCKERMAN, Harriet. The productivity puzzle: Persistence and change in Patterns of publication of Men and women scientists. Advances in Motivation and Achievement, Vol. 2, p. 217-258, Londres, 1984 . Google

CONSELHO NACIONAL DE DESENVOLVIMENTO CIENTÍFICO E TECNOLÓGICO - CNPq. Bolsas individuais no país, RN-028/2015. Acesso em: 10/10/2017. [Visualizar]

COORDENAÇÃO DE APERFEIÇOAMENTO DE PESSOAL DE NÍVEL SUPERIOR - CAPES. Critérios de classificação Qualis - Ensino. Brasília, 2015.

DOERINGER, Peter Brantley; PIORE, Michael Joseph. Internal labor markets and manpower analysis. Sharpe, M., Inc., United States, 1985. Google

FAGERBERG, Jan; SRHOLEC, Martin; VERSPAGEN, Bart. Innovation and economic development. Working Papers on Innovation Studies. Centre for Technology, Innovation and Culture - University of Oslo, Noruega, 2009. Google Ecrosser

GOMES, Joana Aline Vasconcelos. Eficiência do gasto público em educação superior: um estudo sobre as universidades federais do estado de minas gerais. Dissertação (Mestrado em Administração Pública em Rede Nacional - PROFIAP). Universidade Federal de Viçosa. Rio Paranaíba, Minas Gerais - Brasil, 2016. [Visualizar]

GUJARATI, Damodar; PORTER, Dawn. Econometria Básica. AMGH Editora Ltda, $5^{\circ}$ edição, Porto Alegre, 2011. Google

INSTITUTO BRASILEIRO DE GEOGRAFIA E ESTATÍSTICA - IBGE. Síntese de Indicadores Sociais - Uma análise das condições de vida da população brasileira. Rio de Janeiro, 2014.

Indicadores IBGE- Principais destaques da evolução do mercado de trabalho nas regiões metropolitanas abrangidas pela pesquisa. IBGE, 2015.

INSTITUTO DE PESQUISA ECONÔMICA APLICADA - IPEA. Relações universidade-empresa: o papel da infraestrutura pública de pesquisa. Brasília - Ipea, 2003. 
INSTITUTO NACIONAL DE ESTUDOS E PESQUISAS EDUCACIONAIS ANÍSIO TEIXEIRA - INEP. A mulher na educação superior brasileira: 1991-2005. Dados Internacionais de Catalogação na Publicação (CIP), 2007.

KYVIK, Svein; TEIGEN, Mari. Child care, Research Collaboration, and gender diferences in Scientific productivity. Science, Technology, \& Human Values, vol. 21, № 1, p. 54-71, Oslo, 1996. Google Ecrossef

LETA, Jacqueline; LEWISON, Grant. The contribution of women in Brazilian Science: A case study in astronomy, immunology and oceanography. Scientometrics, vol. 57, n 3, p. $339-353,2003$. Google crossref

LIMA, Ricardo. Mercado de trabalho: O capital humano e a teoria da segmentação. Pesquisa Planejamento Econômico, v. 10, n. 1, pp. 217-272. Rio de Janeiro, 1980. [Visualizar] Google

LOUREIRO, Paulo Roberto Amorim. Uma resenha teórica e empírica sobre economia da discriminação. Revista Brasileira de Economia, v. 57, n. 1, pp. 125-157. Rio de Janeiro, 2003. Google Ecrossert

MADALOZZO, Regina. CEOs e Composição do Conselho de Administração: a falta de identificação pode ser motivo para existência de teto de vidro para mulheres no Brasil? Revista RAC, vol. 15, pp. 126-137, Curitiba, 2011. [Visualizar] Google

MINISTÉRIO DA EDUCAÇÃO - MEC. Aspectos conceituais da proposta de reestruturação das carreiras de docentes. Julho, 2012. Acesso em: 01/11/2016. [Visualizar]

Aplicativo para propostas de cursos novos. Manual do usuário, 2015. Acesso em: 11/10/2017. [Visualizar]

MOSCHKOVICH, Marília; ALMEIDA, Ana Maria Fonseca. Desigualdade de Gênero na Carreira Acadêmica no Brasil. Revista de Ciências Sociais, vol. 58, nº 3, Rio de Janeiro, 2015. Google crossref

O GLOBO. Mulheres são minoria em apenas cinco carreiras. Acesso em: 04/11/2016. [Visualizar]

PHELPS, Edmund Strother. The statistical theory of racism and sexism. The American Economic Review, v. 62, n.4, pp. 659-661, 1972. Google

ROEMER, John E. Equality of opportunity. Harvard University Pressa, Cambridge, Massachusetts, London, England, 1998.

SANTOS, Solange Maria dos. O desempenho das universidades brasileiras nos rankings internacionais: áreas de destaque da produção científica brasileira. 2015. 344f. Tese (Doutorado em Ciência da Informação) - Escola de Comunicações e Artes, Universidade de São Paulo, São Paulo, 2015. [Visualizar] 
SANTOS, Cecília MacDowell; IZUMINO, Wânia Pasinato. Violência contra as Mulheres e Violência de Gênero: Notas sobre Estudos Feministas no Brasil. Estudios interdisciplinarios de América Latina y el Caribe (EIAL), v. 16, n.1, 2005. [Visualizar] Google

VELHO, Léa; LEÓN, Elena. A construção social da produção científica por mulheres. Cadernos pagu, vol.10, p. 309-344, 1998. [Visualizar] Google

WAINER, Jacques; VIEIRA, Paula. Avaliação de bolsas de produtividade em pesquisa do CNPq e medidas bibliométricas: correlações para todas as grandes áreas. Perspectivas em Ciência da Informação, v.18, n.2, p.60-78, 2013. [Visualizar] Google 